\title{
A DIRECT VOLUME RENDERING FRAMEWORK FOR THE INTERACTIVE EXPLORATION OF HIGHER-ORDER AND MULTIFIELD DATA
}

\author{
Felix Manke and Burkhard Wünsche \\ Graphics Group, Department of Computer Science, University of Auckland, Private Bag 92019, Auckland, New Zealand \\ fman020@ec.auckland.ac.nz, burkhard@cs.auckland.ac.nz.
}

Keywords: direct volume rendering, visualization tools, vector field visualization, tensor field visualization.

\begin{abstract}
Direct Volume Rendering is a popular method for displaying volumetric data sets without generating intermediate representations. The technique is most frequently applied to scalar data and few specialized techniques exist for visualizing higher-order data, such as tensor fields, directly. This is a serious limitation because progress in medical imaging, satellite technology and numerical simulations has made higher-order and multifield data sets a common entity in medicine, science and engineering.

In this paper we present a framework for the interactive exploration of complex data sets using direct volume rendering. This is achieved by applying sophisticated Software Engineering (SE) to modularize the direct volume rendering pipeline and by exploiting the latest advances in graphics hardware and shading languages to modify rendering effects and to compute derived data sets at runtime.

We discuss how the framework can be used to mimic the latest specialized direct volume rendering algorithms and to interactively explore and gain new insight into high-order and multifield data sets. The capabilities of the framework are demonstrated by three case studies and the efficiency and effectiveness of the framework is evaluated.
\end{abstract}

\section{INTRODUCTION}

The amount and complexity of data from simulations and measurements in science, engineering and medicine is increasing exponentially. Visualization is an essential tool to analyze and explore this overwhelming amount of data and to communicate findings to professionals and laymen.

Traditional visualization tools only offer a fixed number of inbuilt visualization techniques often with specific applications in mind. The need for more flexible approaches to explore data has led to the emergence of powerful visualization toolkits, such as VTK (Schroeder et al., 2000; Kitware, Inc., 2007). These tools allow the user to design their own visualization pipelines using appropriate data sources, data transformation and visualization icons. Visual interfaces can make such tools also accessible for users without programming experience.

A very powerful visualization technique is Direct Volume Rendering (DVR), which was developed for rendering volumetric scalar data sets directly without requiring intermediate representations such as isosurfaces (Levoy, 1990; Westover, 1991). Over recent years the emergence of modern graphics hardware has made interactive DVR on consumer level hardware possible and the technique is now an essential tool in medical imaging and many other scientific and engineering applications.

Direct volume rendering is usually only available as a fixed technique within visualization applications and toolkits and the user has only limited choices to effect the way data is rendered. In particular it is usually not possible to render higher order data such as vector and tensor fields. More specialized techniques have been developed for such applications such as DVR of Line Integral Convolution (LIC) textures, where a 3D texture represents a vector field, or hue balls and lit tensors where tensor information is used to define illumination and color parameters for DVR.

These tailor made techniques are often sufficient 
for one particular application, but so far there are no tools available to give the user full flexibility when exploring volumetric data sets using DVR. For example, existing tools are not designed to use different data sets as input for color and opacity transfer functions (e.g. using anisotropy values derived from tensor data) or to represent different tissue types by different textures.

In this paper we present a framework for the interactive exploration of multifield and higher-order data sets using direct volume rendering, advanced GPU programming and SE design technologies. Section 2 reviews DVR techniques and toolkits supporting it. The identified range of existing applications is used to develop the requirements and motivate the design of our framework. Section 3 and 4 present the design and implementation of our framework, which is evaluated in section 5. Section 6 concludes this paper and discusses issues for future research.

\section{RELATED WORK}

\subsection{Direct Volume Rendering}

Direct volume rendering was developed for transforming scalar volume data directly into an image by associating scalar (density) values with colors and transparencies. Most direct volume rendering algorithms are based on the emission-absorption model where scalar values are interpreted as densities of a gaseous material which emits and absorbs light. An image is created by accumulating the total light intensity for each image pixel which in the simplest case is computed as (Sabella, 1988)

$$
C=\int_{0}^{\infty} c(t) e^{-\int_{0}^{t} \kappa(u) d u} d t
$$

where $t$ is the parameter of a viewing ray through a pixel, $C$ is its color, $c(t)$ the color at the ray parameter $t$ and the integral in the exponent is the total opacity of the ray segment $[0, t]$ which is computed by integrating the particle densities (opacities) along the ray. This equation assumes that at each point density values are associated with color and opacity values which is achieved using color and opacity transfer functions. A detailed discussion of optical models is given in (Max, 1995).

The structure of a volume can be emphasized by using a local illumination function. The most common methods uses the normalized gradients of the scalar field as surface normal (Levoy, 1988). This works well since boundaries between different materials are often characterized by a high density gradient.

\subsection{Advanced Transfer Functions}

Various modifications of the traditional approach to associate scalar voxel values with RGB color and opacity values have been suggested. For example, Spectral Volume Rendering models spectral changes in light caused by interaction with the material within a voxel (Noordmans et al., 2000). The method allows interesting transparency effects and makes it easy to reveal hidden structures in a volume.

Feature rich visualizations can be obtained by replacing one-dimensional transfer functions with multi-dimensional ones and by applying these to scalar or multivariate data (Kniss et al., 2002). Special manipulation widgets make the specification of transfer functions more intuitive and convenient.

Hauser et al. (Hauser et al., 2000; Hauser et al., 2001) devise a technique to fuse DVR, Maximum Intensity Projection (MIP), surface rendering and nonphotorealistic rendering. The authors suggest that this approach is especially useful when visualizing inner structures together with semi-transparent outer layers, similar to the focus-and-context approach known from information visualization. An implementation which allows interactive exploration of volume data sets is also presented.

\subsection{DVR for Higher-Order Data}

The previously surveyed approaches were suitable only to visualize one or multiple scalar data sets. Several authors have presented techniques using DVR to visualize vector and tensor data.

Vector data can be visualized over a 2D surface by encoding the direction of a normalized $3 \mathrm{D}$ vector with a spherical color map which indicates the polar coordinates of the vector (Kindlmann and Weinstein, 1999). Alternatively the vector field direction can be indicated with line segments whose opacity and color encode the vector magnitude and normal component, respectively (Crawfis and Max, 1992).

A more natural representation is to indicate the vector field with a $3 \mathrm{D}$ texture which is then rendered. A popular choice are 3D LIC textures (Cabral and Leedom, 1993). Rezk-Salama et al. present a DVR implementation for interactive exploration of volume LIC (Rezk-Salama et al., 1999). 3D Perception of such volume rendered 3D textures is improved by emphasizing thin thread structures using limb darkening (Helgeland and Andreassen, 2004) or visibilityimpeding halos which indicate depth discontinuities (Interrante and Grosch, 1998; Wenger et al., 2004).

Kindlmann and Weinstein modify the opacity and color transfer functions used in direct volume render- 
ing in order to represent tensor quantities (Kindlmann and Weinstein, 1999). The technique was tailored for the visualization of diffusion tensor fields in the brain.

Various people use textures to visualize diffusion tensor fields, e.g. (Bhalerao and Westin, 2003). The textures can be obtained by integrating streamlines along the maximum diffusion direction within white matter regions which are identified from the mean diffusivities and diffusion anisotropy. Wünsche and Lobb use the diffusion anisotropy to modulate the opacities of white matter tracts and blend it with textures indicating other tissue types. This is achieved by using a classification function which encodes the probability that a field value corresponds to a certain tissue type (Wünsche and Lobb, 2004).

\subsection{Frameworks and Toolkits}

As demonstrated in the previous subsection a limited number of specialized DVR applications exist which are capable of rendering multifield and higher-order data sets and which use multi-dimensional inputs for the color and opacity transfer functions.

Other publications discuss how to implement more general rendering frameworks. Stegmaier et al. present a volume ray casting framework (Stegmaier et al., 2005), which allows to define visualization techniques by implementing fragment shaders. The application is restricted to scalar volume data, for which a gradient can be pre-computed. Other data is not supported and new fields cannot be derived.

Bruckner and Gröller discuss an interactive framework for non-photorealistic volume illustrations (Bruckner and Gröller, 2005). Their system, the VolumeShop, allows to interactively explore and annotate scalar data. Hereby, more than one volume can be rendered simultaneously. Visualization and shading effects are defined by adjusting a two-dimensional transfer function. The application uses hardwareaccelerated rendering and $\mathrm{C}$ for graphics $(\mathrm{Cg})$ as high level shading language.

Teem (Kindlmann, 2003) is a very extensive collection of open-source $\mathrm{C}$ libraries that offer a variety of functionality to process and visualize volumetric data. It supports data of arbitrary dimensionality and lets the user specify which entities to derive and how to map the data to colors and opacities (by defining multi-dimensional transfer functions). However, Teem is not interactive, as it has to be run on command line.

The OpenGL Volumizer is a powerful $\mathrm{C}++$ toolkit for hardware-accelerated high-quality volume renderings and is suitable for very large data sets (Bhaniramka and Demange, 2002) and multi-resolution vol- ume rendering (LaMar et al., 1999). Shaders may be defined that specify the visualization. The OpenGL Volumizer depends on other frameworks such as the Visualization Toolkit (VTK).

VTK is a modular, object-oriented an flexible visualization toolkit (Schroeder et al., 2000; Kitware, Inc., 2007) which incorporates vector and tensor icons and several DVR algorithms. The tools can be extended by overwriting existing classes such as transfer functions. However, it is very difficult to do non-standard applications such as rendering 3D LIC textures and the user has no control which parts of the computation get performed on the graphics card. Users without programming experience can use VTK via a visual programming environment with an integrated self-learning help capability (Telea and van Wijk, 2000).

\section{FRAMEWORK DESIGN}

The direct volume rendering process in the general case can be represented by the pipeline in figure 1 . Taking into account the advanced applications we surveyed in section 2 and the requirements we discussed in the introduction it becomes clear that a flexible design is needed at each stage of the rendering pipeline:

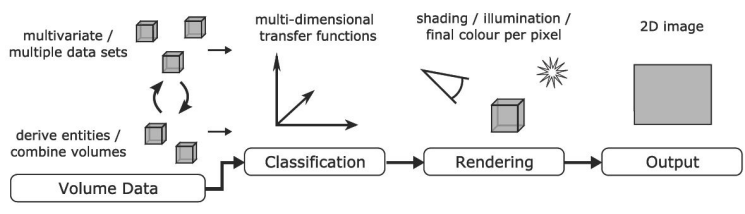

Figure 1: Generalised direct volume rendering pipeline.

- Data initialization stage: It has to be possible to load arbitrary volume data (multiple scalar and higher order data sets). The framework must support the easy integration of arbitrary data types and file formats and it must be possible to derive new entities during run time. Examples are eigenvector fields and textures, such as 3D LIC textures, which subsequently can be used for rendering the data.

- Classification stage: The classification stage must be flexible enough such that the user can use arbitrary components of the data or derived entities to classify the data to be rendered. For example, Diffusion Tensor Imaging data of the brain can be classified by using the mean diffusivity and diffusion anisotropy computed from the diffusion tensor data. Similarly, positron emission tomog- 
raphy (PET) data can be used to determine regions of interest, e.g. a tumor, in an MRI data set.

Data classification does not only involve the use of color and opacity transfer functions, but should also allow the creation of new transfer functions such as texture transfer functions.

- Rendering stage: The rendering can be divided into three components: The reconstruction technique, the type of DVR algorithm (see subsection 3.4) and the desired rendering effects. The selected techniques affect both rendering speed and image quality.

Optimal reconstruction functions for specific application areas such as volume rendering are an ongoing topic of research (e.g. (Marschner and Lobb, 1994; Moorhead II and Zhu, 1995; Möller et al., 1998; Mueller et al., 1999)). Reconstruction of vector and tensor data is more complicated and can be achieved using spectral analysis (Aldroubi and Basser, 1999). Note that when using texture based DVR algorithms usually a bi- or trilinear reconstruction filter is used.

By controlling rendering effects, the user can emphasize different aspects of the data and improve the visual perception of features. Examples are gradient based shading, color mapping of curvature, and emphasis of the silhouette boundary. Also the user might want to switch interactively between rendering different aspects of a data set, e.g. Computed Tomography (CT) data alone and combined with PET data.

\subsection{Run-Time Modification of Rendering Effects and Data Sets}

The most important user-specific aspects of the rendering framework are the derivation of new fields, the processing of the data (e.g. reconstruction), and the specification of visualization techniques and shading effects.

At this stage advanced reconstruction kernels have not been implemented, but they can be incorporated using the techniques described in the following sections.

\subsubsection{Derivation of New Fields}

The derivation of new entities is to be done on the GPU in order to achieve efficiency and to minimize data transfer time. We call the $\mathrm{Cg}$ code blocks or modules that implement this derivation operators. The main input of an operator are volume data sets and the output is a texture object that holds the derived values. Note that, by specifying operators in the CgFX syntax, the full capabilities of the graphics hardware is available for the execution (vertex and fragment shaders, multiple render targets, etc.).

Loaded data is exclusively used by operators and visualization effects, which both run on the GPU and therefore will be implemented by the user as $\mathrm{Cg}$ shader programs. Hence, to facilitate a practicable work with the framework, we keep the specification of resources and the fragment-shader definition at the same location.

An even more powerful representation could be achieved by adopting the generalized field data structure we previously presented in (Wünsche, 2002). The data structure is based on arithmetic trees allows lazy evaluation of derived fields and the combination of structured, unstructured and analytic data.

\subsubsection{Rendering Effects}

By analyzing existing DVR applications we observed that the executed $\mathrm{Cg}$ shader code can be separated into code that is specific for a particular DVR algorithm (written by developers) and code that is specific to a user-defined rendering effect (written by the user and which might have to be changed at run-time).

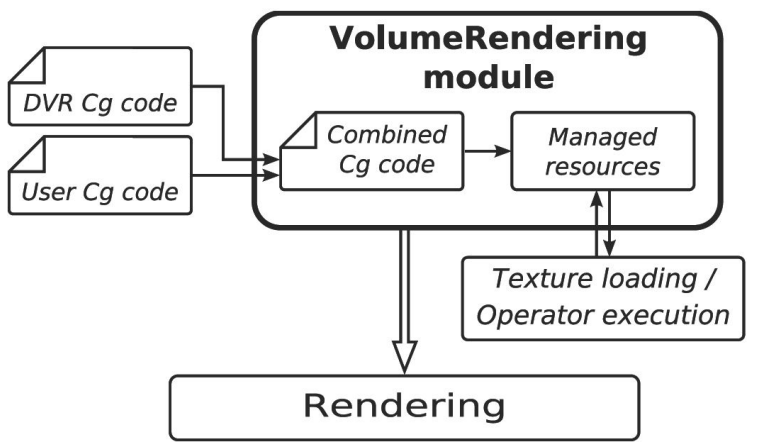

Figure 2: The volume rendering module that manages the $\mathrm{Cg}$ code and resources.

These two code sections must hence be physically and conceptually separated. Figure 2 shows that our framework contains a module that assembles the DVR algorithm's and the users Cg code and manages the specified resources (volume data sets or textures). During the initialization, the compiled $\mathrm{Cg}$ code is analyzed using the $\mathrm{Cg}$ Core Runtime API, data sets are loaded and operators executed. By making use of $\mathrm{Cg}$ interfaces, the implementation of a rendering effect (which we call evaluator) is held abstract and the user can define as many different evaluators as desired using several implementations of the abstract interface. The volume rendering module makes interactive switching of evaluators possible. 


\subsection{Extendability}

The framework must be flexible enough to allow developers to integrate new data formats and to implement new DVR algorithms. A unified scheme that makes extending the framework as straightforward as possible is obtained by using a generic template factory that lets developers register new implementations in a single line of code. After registration, a sub-class can be instantiated throughout the application using a unique identifier, without the need of knowing the concrete data type.

In order to easily initialize objects at start-up, we have developed a unified design for initializing all possible state variables. Two fundamental problems arise when dealing with state variables of unknown objects (as they may be present in the framework due to sub-classing by other developers): The state variables themselves are unknown (i.e. their "name" or signature) and their data type may differ.

To be able to initialize the state variables of the unknown objects we introduce a design which we call parameter design pattern: Every pair of Get... and Set... methods is encapsulated by a "parameter" object that hides the data type of the state variable using a string representation, which is used by the application.

With these two concepts, the generic factory and the parameter design pattern, we are able to automatically instantiate and initialize new implementations. Note that if a data set has more than four components per voxel, a single volume data set must be associated with multiple texture sampler objects. The framework supports a splitting of the data into several textures and samplers.

\subsection{Framework Components}

The main components of our rendering framework are shown in figure 3 . Besides the concepts and modules discussed so far, the framework contains a controller object that manages the entire program execution (initialization, rendering and termination). During the start, a configuration file is parsed. It contains settings that specify global states of the application. Further on, a renderer is introduced to render all graphical objects that are registered. It also updates the camera according to the user input.

\subsection{GPU Acceleration}

In order to achieve real-time performance we use hardware-accelerated direct volume rendering methods which use the data processing model of the graph-

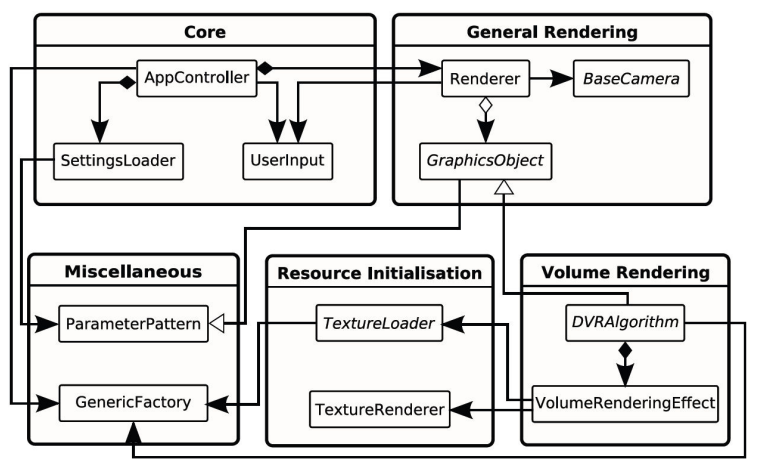

Figure 3: The main components of the direct volume rendering framework. Note that the VolumeRenderingEffect component combines $\mathrm{Cg}$ shaders and manages specified resources.

ics pipeline shown in figure 4 . The model was originally developed for fast rendering of geometric primitives defined by vertices, such as lines and polygons. The simplified representation in the image concentrates on the components relevant to our research.

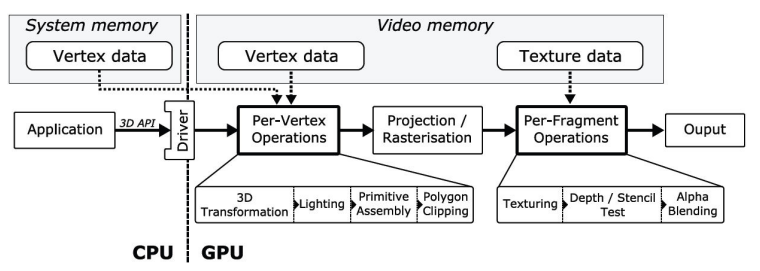

Figure 4: Simplified model of the general graphics pipeline.

The volume rendering equation 1 can be solved on the GPU by using object-order algorithms which project the volume data onto the image plane using the texture mapping and blending capabilities of the graphics hardware. Since graphics cards do not support volumetric rendering primitives a polygonal proxy geometry, usually axis aligned or view aligned slices, is used (Rezk-Salama et al., 2000).

In order to avoid problems with perspective projection when using object-aligned slices we perform a perspective correction per viewing ray in a fragment shader. In this case the position of the vertices has to be passed to the fragment shader. This is done by a simple vertex shader program (because vertex shader outputs are interpolated during rasterization, the fragment shader inputs correspond to the pixel positions) (Manke, 2007).

Object-order approaches have the disadvantage that a significant amount of voxels are processed which might not contribute to the final image. Raycasting makes it possible to skip transparent regions, adjust step size according to the importance of a re- 
gion and to utilize predictions whether the currently processed fragment will be occluded by a later processed slice. GPU-supported ray-casting, introduced by (Krüger and Westermann, 2003; Röttger et al., 2003), uses the one-to-one correspondence between pixels of the resulting image and the traced viewing rays. Hence it is straightforward to encode the rays in 2D textures (start point and direction). Additional data such as the accumulated colors and opacities are also stored in textures.

\section{IMPLEMENTATION}

In our implementation we use as shading language $\mathrm{Cg}$ (C for Graphics) since HLSL (High Level Shading Language) is only available in conjunction with the Microsoft Direct3D API, and GLSL (OpenGL Shading Language) lacks some advanced features required for achieving modularity. In particular $\mathrm{Cg}$ provides interfaces for compile-time polymorphism, \# include pre-processor directive which we use to integrate user-defined $\mathrm{Cg}$ code into the rendering framework, and CgFX Files, semantics, and annotations. A detailed discussion of the features and data types used and differences to other shading languages is given in (Manke, 2007).

Basic mathematics for 3D graphics is provided by Graphics $3 D$ (McGuire, 2007) which has the additional advantage that it wraps the OpenGL API and provides an object-oriented rendering framework. Furthermore, the Extensible Markup Language (XML) is used to specify the settings of the application. A simple and minimalist open-source library, TinyXml (Thomason, 2007), is used to load and parse the XML fies.

\section{RESULTS}

To demonstrate the effectiveness and flexibility of our framework and the discussed concepts, we developed a prototype of the framework. Within it we implemented two DVR algorithms and support for different data formats.

In three case studies we show how to define resources, operators and evaluators. At first, we used a scalar CT data set of the head of the Visible Male (acquired by (National Library of Medicine, NIH, 2007), downloaded from (Röttger, 2006)). Renderings of different evaluators are shown in figure 5.

Next we used a CT and a PET data set of a monkey (acquired by (Laboratory of Neuro Imaging, UCLA,
2007), downloaded from (Röttger, 2006)). Our framework makes it possible to load and render these data sets separately and simultaneously as demonstrated in figure 6 .

In the third case study we procedurally computed a vector field on the GPU using an operator. As shown in figure 7, two evaluators were used to visualize either the vector field encoded as RGB colors or using real-time 3D Line Integral Convolution (adopted from (Lakshmanan, 2006)).

The renderings for all case studies were performed at interactive speeds. We used a machine with an Intel Pentium 3.4 GHz CPU, 2.0 GB main memory and an NVIDIA Quadro FX 3400 with $256 \mathrm{MB}$ video memory. The set-up time depends on texture loaders and operators. For the first two case studies the set up required between 0.25 and 0.55 seconds and rendering between 30 and $90 \mathrm{~ms}$ per frame (33 to 11 frames/second). In the LIC case study, the setup required 0.8 seconds and the rendering $225 \mathrm{~ms}$ per frame (4.4 frames/second) on average.

The full report of the implementation (Manke, 2007) demonstrates that the user defined source code is well structured and short (between two and 16 lines per evaluator), because the Cg code for the DVR algorithm is separated.

\section{CONCLUSION}

We have presented a modular and flexible framework for interactive direct volume rendering of complex data sets. The framework is entirely GPU-based and can be easily extended by developers.

In contrast to existing applications and toolkits it is completely modular and the user can interactively derive new entities and modify rendering and shading effects to explore complex data sets. We use advanced mechanisms of the $\mathrm{Cg}$ language to provide a flexibility that is usually difficult to achieve on the specialized and restrictive graphics hardware.

In the future, we will add additional functionalities such as volume clipping, higher quality rendering algorithms, and flexible reconstruction filters. Also, multi-resolution techniques will be implemented for handling large data sets. We are currently conducting more complex case studies which involve vector and tensor data sets, e.g. DW-MRI data and we are using our framework to develop texture transfer functions.

Finally we want to make the framework more user-friendly and provide a graphical user interface which offers menus and dialogs for loading data, selecting shaders and effects and loading and developing new ones. Examples are formula editors with GUI 

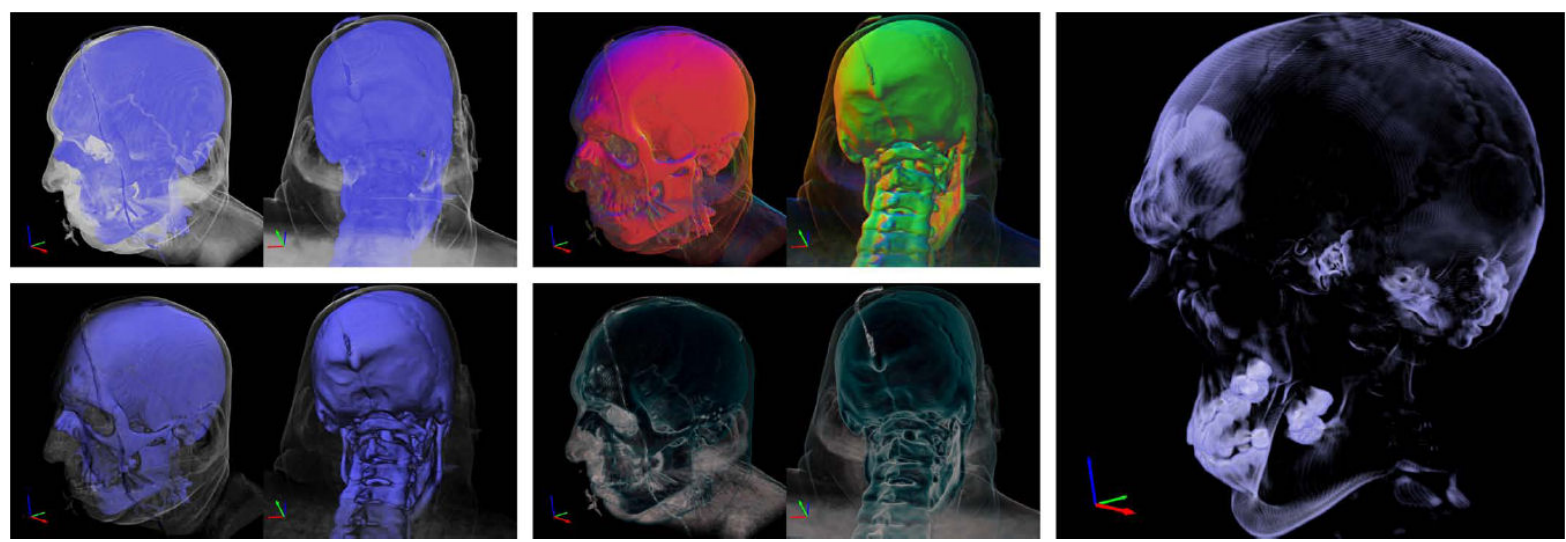

Figure 5: Different renderings of a CT data set. Top-left: Evaluation of a basic 1D transfer function. Bottom-left: Additional diffuse lighting. Top-center: Gradient shading that shows the direction of the gradient vectors. Bottom-center: Artistic shading that enhances the silhouette of rendered structures. Right: $2 \mathrm{D}$ transfer function using the scalar data value and gradient magnitude.

for deriving new data sets, spreadsheet like interfaces for interactively exploring and comparing the effects of different shaders, and a visual programming interface based on the data flow paradigm for modifying the DVR pipeline.
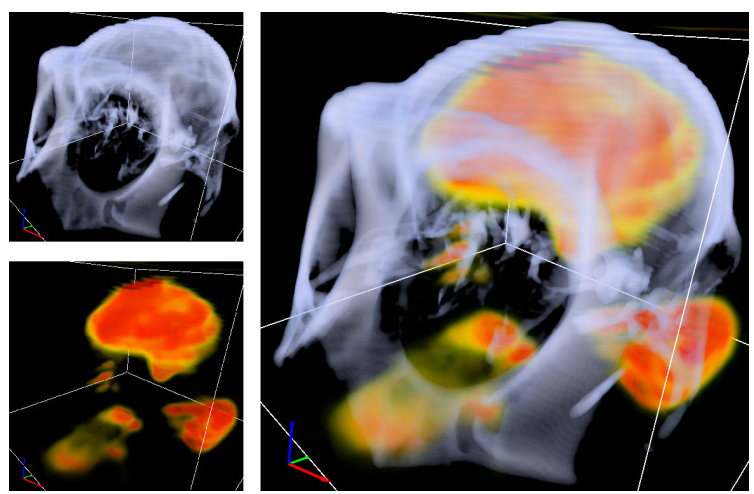

Figure 6: Combined rendering (right) of a CT (top-left) and a PET (bottom-left) data set of a monkey brain.
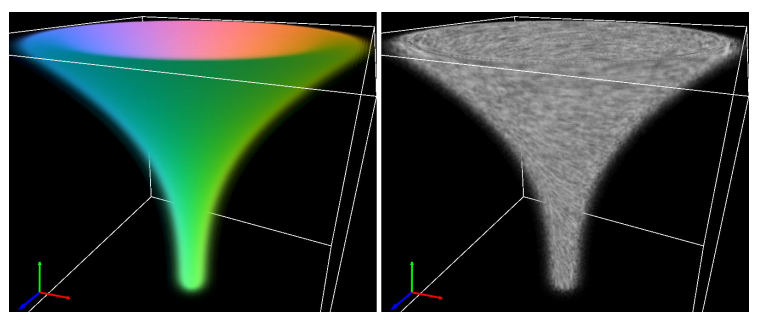

Figure 7: Renderings of the LIC case study. Left: Color encoded procedural vector field. Right: Interactive 3D LIC rendering. The opacity is inversely proportional to the vector length.

\section{REFERENCES}

Aldroubi, A. and Basser, P. J. (1999). Reconstruction of vector and tensor fields from sampled discrete data. In The Functional and Harmonic Analysis of Wavelets and Frames, volume 247 of Contemporary Mathematics, pages 1-15. American Mathematical Society.

Bhalerao, A. and Westin, C.-F. (2003). Tensor splats: Visualising tensor fields by texture mapped volume rendering. In Proceedings of MICCAI'03, pages 294-901.

Bhaniramka, P. and Demange, Y. (2002). OpenGL volumizer: a toolkit for high quality volume rendering of large data sets. In Proceedings of the 2002 IEEE Symposium on Volume Visualization and Graphics, pages 45-54, Piscataway, NJ, USA. IEEE Press.

Bruckner, S. and Gröller, M. E. (2005). VolumeShop: An interactive system for direct volume illustration. In Proc. of IEEE Visualization 2005, pages 671-678.

Cabral, B. and Leedom, L. C. (1993). Imaging vector fields using line integral convolution. In Proceedings of SIGGRAPH '93, pages 263-272. ACM SIGGRAPH.

Crawfis, R. and Max, N. (1992). Direct volume visualization of three-dimensional vector fields. In Workshop on Volume Visualization, pages 55-60. ACM Press.

Hauser, H., Mroz, L., Bischi, G.-I., and Gröller, M. E. (2000). Two-level volume rendering - fusing MIP and DVR. In Proceedings of Visualization 2000, pages 211-218. IEEE Press.

Hauser, H., Mroz, L., Bischi, G. I., and Gröller, M. E. (2001). Two-level volume rendering. IEEE Transactions on Visualization and Computer Graphics, 7(3):242-252.

Helgeland, A. and Andreassen, O. (2004). Visualization of vector fields using seed LIC and volume rendering. IEEE Transactions on Visualization and Computer Graphics, 10(6):673-682. 
Interrante, V. and Grosch, C. (1998). Visualizing 3D flow. IEEE Computer Graphics and Applications, 18(4):49-53.

Kindlmann, G. (2003). Teem: Tools to process and visualize scientific data and images. URL: http://teem.sourceforge.net.

Kindlmann, G. and Weinstein, D. (1999). Hue-balls and littensors for direct volume rendering of diffusion tensor fields. In Proceedings of Visualization '99, pages 183189. IEEE Press.

Kitware, Inc. (2007). VTK Home Page. URL: http://public.kitware.com/VTK.

Kniss, J., Kindlmann, G., and Hansen, C. (2002). Multidimensional transfer functions for interactive volume rendering. IEEE Transactions on Visualization and Computer Graphics, 8(3):270-285.

Krüger, J. and Westermann, R. (2003). Acceleration techniques for GPU-based volume rendering. In Proc. of Visualization 2003, pages 287-292. IEEE Press.

Laboratory of Neuro Imaging, UCLA (2007). Monkey atlas. URL: http://www.loni.ucla.edu/ [Last accessed 21/09/2007]

Lakshmanan, V. (2006). Interactive 3D line integral convolution on the GPU. Master's thesis, School of Electrical Engineering and Computer Science, Oregon State University, Corvallis, OR, USA.

LaMar, E., Hamann, B., and Joy, K. I. (1999). Multiresolution techniques for interactive texture-based volume visualization. In Proceedings of Visualization '99, pages 355-361. IEEE Press.

Levoy, M. (1988). Display of surfaces from volume data. IEEE Comp. Graphics \& Applications, 8(5):29-37.

Levoy, M. (1990). Efficient ray tracing of volume data. ACM Transactions on Graphics, 9(3):245-261.

Manke, F. (2007). A modular GPU-based direct volume renderer for visualising multi-dimensional highorder data. 780 project report, Dept. of Computer Science, University of Auckland, Auckland, New Zealand. URL: http://www.cs.auckland.ac.nz/ oburkhard/Reports/2007_S1_FelixManke.pdf.

Marschner, S. R. and Lobb, R. J. (1994). An evaluation of reconstruction filters for volume rendering. In Proc. of Visualization '94, pages 100-107. IEEE Press.

Max, N. (1995). Optical models for direct volume rendering. IEEE Transactions on Visualization and Computer Graphics, 1(2):99-108.

McGuire, M. (2007). G3d engine. URL: http://g3dcpp.sourceforge.net [Last accessed 24/08/2007].

Möller, T., Müller, K., Kurzion, Y., Machiraju, R., and Yagel, R. (1998). Design of accurate and smooth filters for function and derivative reconstruction. In Proceedings of the 1998 Symposium on Volume Visualization (VOLVIS-98)), pages 134-151. ACM Press.

Moorhead II, R. J. and Zhu, Z. (1995). Signal processing aspects of scientific visualization. IEEE Signal Processing Magazine, 12(5):20-41.
Mueller, K., Möller, T., and Crawfis, R. (1999). Splatting without the blur. In Proceedings of Visualization '99, pages 363-370. IEEE Press.

National Library of Medicine, NIH (2007). The visible human project. URL: http://www.nlm.nih.gov/re search/visible/visible_human.html.

Noordmans, H. J., van der Voort, H. T. M., and Smeulders, A. W. M. (2000). Spectral volume rendering. IEEE Transactions on Visualization and Computer Graphics, 6(3):196-207.

Rezk-Salama, C., Engel, K., Bauer, M., Greiner, G., and Ertl, T. (2000). Interactive volume rendering on standard PC graphics hardware using multitextures and multi-stage rasterization. In Proc. SIGGRAPH/Eurographics Graphics Hardware Workshop 2000, pages 109-118.

Rezk-Salama, C., Hastreiter, P., Teitzel, C., and Ertl, T. (1999). Interactive exploration of volume line integral convolution based on 3D-texture mapping. In Proc. of Visualization '99, pages 233-240. IEEE Press.

Röttger, S. (2006). The volume library. URL: http:// www9.informatik.uni-erlangen.de/External/vollib.

Röttger, S., Guthe, S., Weiskopf, D., Ertl, T., and Strasser, W. (2003). Smart hardware-accelerated volume rendering. In Proceedings of the Symposium on Data Visualisation 2003 (VISSYM '03), pages 231-238.

Sabella, P. (1988). A rendering algorithm for visualizing 3D scalar fields. In Proceedings of SIGGRAPH '88, pages 51-58. ACM Press.

Schroeder, W. J., Avila, L. S., and Hoffman, W. (2000). Visualizing with VTK: A tutorial. IEEE Computer Graphics and Applications, 20(5):20-27.

Stegmaier, S., Strengert, M., Klein, T., and Ertl, T. (2005). A simple and flexible volume rendering framework for graphics-hardware based raycasting. In Proceedings of the International Workshop on Volume Graphics '05, pages 187-195.

Telea, A. and van Wijk, J. J. (2000). SMARTLINK: an agent for supporting dataflow application construction. In Proceedings of the Joint EUROGRAPHICS and IEEE TCVG Symposium on Visualization, pages 189-198, Amsterdam, Netherlands, May 29-31 2000.

Thomason, L. (2007). Tinyxml. URL: http://sourceforge .net/projects/tinyxml/ [Last accessed 24/08/2007].

Wenger, A., Keefe, D. F., Wenger, S. Z. A., Keefe, D. F., and Laidlaw, D. H. (2004). Volume rendering of thin thread structures within multivalued scientific data sets. IEEE Transactions on Visualization and Computer Graphics, 10(6):664-672.

Westover, L. A. (1991). SPLATTING: A Parallel, FeedForward Volume Rendering Algorithm. PhD thesis, University of North Carolina, Chapel Hill, NC.

Wünsche, B. C. (2002). A field data structure for improved interactive exploration of scientific data sets. In Proceedings of IVCNZ '02, pages 13-18.

Wünsche, B. C. and Lobb, R. (2004). The 3d visualization of brain anatomy from diffusion-weighted magnetic resonance imaging data. In Proceedings of GRAPHITE 2004, pages 74-83. ACM Press. 